\title{
Does use of silane-containing universal adhesive eliminate the need for silane application in direct composite repair?
}

\author{
Carolina Lopes da SILVA(a) \\ Maitê Munhoz SCHERER(b) \\ Laura Teixeira MENDES(b) \\ Luciano CASAGRANDE(b) iD \\ Vicente Castelo Branco \\ LEITUNE(c) \\ Tathiane Larissa LENZI ${ }^{(b)}$ \\ (a) Universidade Federal do Rio Grande do \\ Sul - UFRS, School of Dentistry, Department \\ of Surgery and Orthopedics, Porto Alegre, \\ RS, Brazil. \\ (b) Universidade Federal do Rio Grande do Sul \\ - UFRS, School of Dentistry, Post-Graduate \\ Program in Pediatric Dentistry, Porto Alegre, \\ RS, Brazil. \\ (c) Universidade Federal do Rio Grande do \\ Sul - UFRS, School of Dentistry, Dental \\ Materials Laboratory, Porto Alegre, \\ RS, Brazil.
}

Declaration of Interests: The authors certify that they have no commercial or associative interest that represents a conflict of interest in connection with the manuscript.

Corresponding Author:

Tathiane Larissa Lenzi

E-mail: tathilenzi@hotmail.com

https://doi.org/10.1590/1807-3107bor-2020.vol34.0045

Submitted: October 24, 2019

Accepted for publication: March 30, 2020

Last revision: April 15, 2020

\begin{abstract}
This in vitro study aimed to evaluate the effect of a silane-containing universal adhesive used with or without a silane agent on the repair bond strength between aged and new composites. Forty nanohybrid composite resin blocks were stored in distilled water for 14 $\mathrm{d}$ and thermo-cycled. Sandpaper ground, etched, and rinsed speciments were randomly assigned into four experimental groups: silane + two-step etch-and-rinse adhesive system, two-step etch-and-rinse adhesive system, silane + silane-containing universal adhesive system, and silane-containing universal adhesive system. Blocks were repaired using the same composite. After $24 \mathrm{~h}$ of water storage, the blocks were sectioned and bonded sticks were submitted to microtensile testing. Ten unaged, non-repaired composite blocks were used as a reference group to evaluate the cohesive strength of the composite. Two-way ANOVA and Tukey's tests were used to analyze average $\mu$ TBS. One-way ANOVA and Dunnet post-hoc tests were used to compare the cohesive strength values and bond strength obtained in the repaired groups $(\alpha=0.05)$. The $\mu \mathrm{TBS}$ values were higher for the silane-containing universal adhesive compared to the two-step etch-and-rinse adhesive system $(p=0.002)$. Silane application improved the repair bond strength $(p=0.03)$. The repair bond strength ranged from 39.3 to $65.8 \%$ of the cohesive strength of the reference group. Using universal silane-containing adhesive improved the repair bond strength of composite resin compared to two-step etch-and-rinse adhesive. However, it still required prior application of a silane agent for best direct composite resin repair outcomes.
\end{abstract}

Keywords: Tensile Strength; Dental Restoration Repair; Dental Restoration, Permanent.

\section{Introduction}

The annual failure rate of composite resin restorations varies from 1 to $5 \%{ }^{1}$ in permanent teeth and 1.7 to $12.9 \%^{2}$ in primary dentition. Basically, the management of defective restorations includes two options: replacement or repair of the restoration. ${ }^{3}$ Although clinical criteria for evaluating direct restorations have been proposed, ${ }^{4}$ it is not clear which clinical situations one must choose repair or replacement of defective restorations. ${ }^{5,6}$ The general consensus tends toward restoration repair given its numerous advantages, such as preservation of dental structure and reduction of 
treatment costs. ${ }^{3}$ On the other hand, re-restoring teeth can require more complex restorative procedures that raise the risk of initiating a re-intervention cascade, known as the restorative cycle. ${ }^{7}$ Although most dentists claim to perform repairs, and this approach has been adopted by most dental schools, the proportion of truly repaired restorations is still low. ${ }^{8}$ Repair may increase the survival of restorations placed in primary and permanent teeth, ${ }^{9,10}$ but there is no gold standard protocol or materials established for treating aged composite surfaces before repair. ${ }^{11}$

Successful repair procedures require a durable bond between the old restoration and the new composite resin. New composite may be adhered to aged composite through micromechanical interlocking to irregularities in the prepared surface and through chemical bonding to the filler particles and the organic matrix. . $^{12,13,14}$ Bonding agents improve the chemical bonds between the old and new materials. Silanes increase surface wetting, thereby enhancing diffusion of the bonding agent into the substrate. ${ }^{15}$ Silane coupling agents may also form covalent bonds with filler particles and co-polymerize with the methacrylate groups present in repair material. ${ }^{16} \mathrm{~A}$ recent systematic review showed that application of silane coupling agents and adhesives play a role in improving repair bond strength when physical treatments, such as burs, were applied to the aged composite. ${ }^{12}$
Silane agents have been directly incorporated into adhesive systems. Use of a silane-containing universal adhesive could eliminate the need to apply silane as a separate step during the clinical protocol for composite repair. Evidence about effect of a silane-containing universal adhesive associated or not with a silane agent on the repair bond strength of composite is limited and the results are contradictory. ${ }^{17,18}$ Therefore, the current study sought to evaluate the effect of a silane-containing universal adhesive used with or without a silane agent on the repair bond strength between aged and new composites. The hypothesis was that use of a silane-containing universal adhesive would eliminate the silane application for direct composite repair.

\section{Methodology}

A silane coupling agent (RelyX Ceramic Primer, 3M Oral Care, St. Paul, USA) and two adhesive systems were tested: a two-step etch-and-rinse adhesive system (Adper Single Bond Plus, 3M Oral Care, St. Paul, USA) and a silane-containing universal adhesive system (Scotchbond Universal Adhesive, 3M Oral Care, St. Paul, USA). A1E and A3B shades of the nanohybrid composite resin (Filtek Z350 XT, 3M Oral Care, St. Paul, USA) were used in order to differentiate between the aged and new composite resin. A detailed description of the materials is presented in Table 1.

Table 1. Composition and application mode of the materials tested.

\begin{tabular}{|c|c|c|}
\hline $\begin{array}{l}\text { RelyX Ceramic Primer } \\
\text { (3M Oral Care, St. Paul,USA) } \\
\text { \#Batch number } 1720700505\end{array}$ & $\begin{array}{c}\text { Methacryloxypropyl trimethoxysilane; water; ethyl alcohol } \\
\text { 3-(trimetoxysilyl methacrylate) }\end{array}$ & $\begin{array}{l}\text { Apply one coat of silane } \\
\text { Gently air dry for } 5 \mathrm{~s}\end{array}$ \\
\hline $\begin{array}{l}\text { Adper Single Bond Plus } \\
\text { (3M Oral Care, St. Paul, USA) }\end{array}$ & Etchant : $37 \%$ phosphoric acid & $\begin{array}{c}\text { Apply } 2 \text { consecutive coats of adhesive } \\
\text { for } 15 \mathrm{~s} \text { with gentle agitation }\end{array}$ \\
\hline \#Batch number 1812300361 & $\begin{array}{c}\text { HEMA, water, ethanol, Bis-GMA, dimethacrylates, amines, } \\
\text { metacrylate-functional copolymer of polyacrylic and } \\
\text { polyitaconic acids, } 10 \% \text { by weight of } 5 \text { nanometer-diameter } \\
\text { spherical silica particles }\end{array}$ & $\begin{array}{l}\text { Gently air dry for } 5 \mathrm{~s} \\
\text { Light-cure for } 10 \mathrm{~s}\end{array}$ \\
\hline $\begin{array}{l}\text { Z350 XT A1E e A3B Shades } \\
\text { (3M Oral Care, St. Paul,, USA) }\end{array}$ & $\begin{array}{l}\text { Bis-GMA, UDMA, TEGDMA, Bis-EMA, non-agglomerated/ } \\
\text { non-aggregated } 20 \mathrm{~nm} \text { silica filler, non-agglomerated/ }\end{array}$ & Insert the composite in $2 \mathrm{~mm}$ increments \\
\hline $\begin{array}{l}\text { \#Batch numbers } 1729300455 \text {, } \\
1732800739\end{array}$ & $\begin{array}{c}\text { non-aggregated } 4 \text { to } 11 \mathrm{~nm} \text { zirconia filler, and aggregated } \\
\text { zirconia/silica cluster filler }\end{array}$ & Light-cure for $20 \mathrm{~s}$ \\
\hline
\end{tabular}

MDP: 10-methacryloyloxydecyl-dihydrogen-phosphate; Bis-GMA: bisphenyl-glycidyl methacrylate; HEMA: 2-hydroxyethyl methacrylate;

TEGDMA: triethylene glycol dimethacrylate; Bis-EMA: ethoxylated bisphenol-A dimethacrylate; UDMA: urethane dimethacrylate 


\section{Preparation of aged composite blocks}

Forty blocks of nanohybrid composite resin (A1E shade) measuring $8 \times 8 \mathrm{~mm}$ in depth and width and $4 \mathrm{~mm}$ in height were fabricated using a metallic mold $(8 \times 8 \times 8 \mathrm{~mm})$. The mold was fixed on a glass slab. Composite resin was packed into the mold in two increments that were each light cured for $20 \mathrm{~s}$ with a light-emitting diode curing unit (Radii-cal; SDI, Victoria, AUS) with a light output of at least $1,250 \mathrm{~mW} / \mathrm{cm}^{2}$. Light intensity output was monitored with a Demetron Curing Radiometer (Kerr, Orange, USA). The composite was carefully condensed with a clean filling instrument in order to avoid contamination and void entrapment. After setting, composites were gently removed from the mold and the thickness of each block was confirmed with a digital caliper (Absolute Digimatic, Mitutoyo, Tokyo, Japan). The specimens were stored in distilled water at $37^{\circ} \mathrm{C}$ for $14 \mathrm{~d}^{19}$ prior to aging. The blocks were further aged by thermal cycling 5,000 times between 5 and $55^{\circ} \mathrm{C}$, with a dwell time of $20 \mathrm{~s}$ and transfer time of $3 \mathrm{~s} .{ }^{19}$ The aged specimen surfaces were wet-ground with 320-grit silicon carbide grinding paper for $5 \mathrm{~s}$ to remove the superficial resin-rich layer and create standardized repair surfaces. ${ }^{19,20}$ All specimen surfaces were then etched with $37 \%$ phosphoric acid for $30 \mathrm{~s}$, washed with air/water spray for $60 \mathrm{~s}$, and dried with a blast of air for $60 \mathrm{s.}{ }^{18}$

\section{Bonding procedures}

The 40 aged blocks were randomly assigned (Random Allocation software, version 1.0, Iran) into four experimental repair protocol groups $(n=10)$ : silane + two-step etch-and-rinse adhesive system, two-step etch-and-rinse adhesive system, silane + silane-containing universal adhesive system, and silane-containing universal adhesive system. All materials were applied according to the manufacturer's recommendations (Table 1). The aged composite blocks were carefully placed over the original mold and then repaired using nanohybrid composite resin (A3B shade). Resin was applied in two incremental layers that were each light cured for $20 \mathrm{~s}$ following the same protocol as the aged specimens. This process resulted in 8-mm high specimens. Upon removal from the mold, the specimen surfaces covered by the mold were further cured for $20 \mathrm{~s}$. Specimens were stored in distilled water at $37^{\circ} \mathrm{C}$ for $24 \mathrm{~h}$. A single trained operator carried out all procedures.

\section{Microtensile bond strength ( $\mu$ TBS)}

To guarantee that the testing machine operator was blinded, each composite block was numbered according to the randomization sequence. Blocks were sectioned into sticks with a cross-sectional area of approximately $0.8 \mathrm{~mm}^{2}$ using a water-cooled diamond saw in a cutting machine (Isomet, Buehler, Lake Bluff, USA). Approximately 40 sticks were obtained for each block. The sticks were carefully examined with a stereomicroscope (HMV-2, Shimadzu Corp., Kyoto, Japan) at 40× magnification. Those with interfacial flaws, gaps, bubbles, or other defects were discarded. The cross-sectional area of each stick was measured with a digital caliper (Absolute Digimatic, Mitutoyo, Tokyo, Japan) to calculate the bond strength values, measured in MPa. Pretesting failures were not observed. The bonded sticks were attached to a universal testing machine for microtensile testing (EZ-SX series, Shimadzu Corp., Kyoto, Japan) with cyanoacrylate and tested at a crosshead speed of $1 \mathrm{~mm} / \mathrm{min}$. The $\mu \mathrm{TBS}$, measured in MPa, was obtained by dividing the load at failure $(\mathrm{N})$ by the cross-sectional area $\left(\mathrm{mm}^{2}\right)$ of each stick.

\section{Failure mode}

The fracture surfaces were examined under a stereomicroscope (HMV-2, Shimadzu Corp., Kyoto, Japan) at $40 \times$ magnification to determine whether the failure mode was adhesive (failure between restorative material and bonding agent or between bonding agent and repair composite) or cohesive (failure exclusively within the aged or new composite resin). A examiner blind to experimental groups evaluated the failure mode.

\section{Cohesive strength of non-aged composite - reference group}

Ten blocks of nanohybrid composite resin (A1E shade) measuring $8 \times 8 \times 8 \mathrm{~mm}$ were fabricated using a metallic mold. The mold was fixed on a glass slab. Composite was packed into the mold in four increments that were each light cured for $20 \mathrm{~s}$ with 
a light-emitting diode curing unit (Radii-cal; SDI, Victoria, AUS). After inserting the last increment, the Mylar strip was pressed down over the mold for $30 \mathrm{~s}$ and the specimen was light cured through the strip. The thickness of each specimen was verified with a digital caliper. Specimens were stored in distilled water at $37^{\circ} \mathrm{C}$ for $24 \mathrm{~h}$ then prepared for the $\mu \mathrm{TBS}$ test. Figure summarizes the experimental design.

\section{Statistical analysis}

The experimental unit was the resin block. Thus, the $\mu$ TBS values from every stick from the same block were averaged for statistical analysis. Sticks with cohesive failures from the repaired groups were not included in the analysis. The $\mu$ TBS mean for each test group represents the mean of the ten blocks used per group. The ten blocks sample size was previously estimated using the following parameters: $80 \%$ power, a coefficient of variation of $20 \%$, and assuming a two-sided 5\% significance level for comparisons.
Normal data distribution was confirmed using a Kolmogorov-Smirnov test. The $\mu$ TBS means of the repaired groups were analyzed by two-way ANOVA and Tukey's post-hoc tests. One-way ANOVA and Dunnet post-hoc tests were used to compare the cohesive strength values and bond strength obtained in the repaired groups. The significance level was set at $p<0.05$. Statistical analyses were performed using Minitab18 software (Minitab Inc., State College, USA).

\section{Results}

The $\mu$ TBS means, standard deviations, and distribution of the failure mode for all experimental groups are shown in Table 2. Cohesive strength values of non-aged specimens were higher than bond strength obtained in all repaired groups ( $p<0.01$ ). Repair bond strength ranged of $39.3 \%$ to $65.8 \%$ of cohesive strength of the reference group.
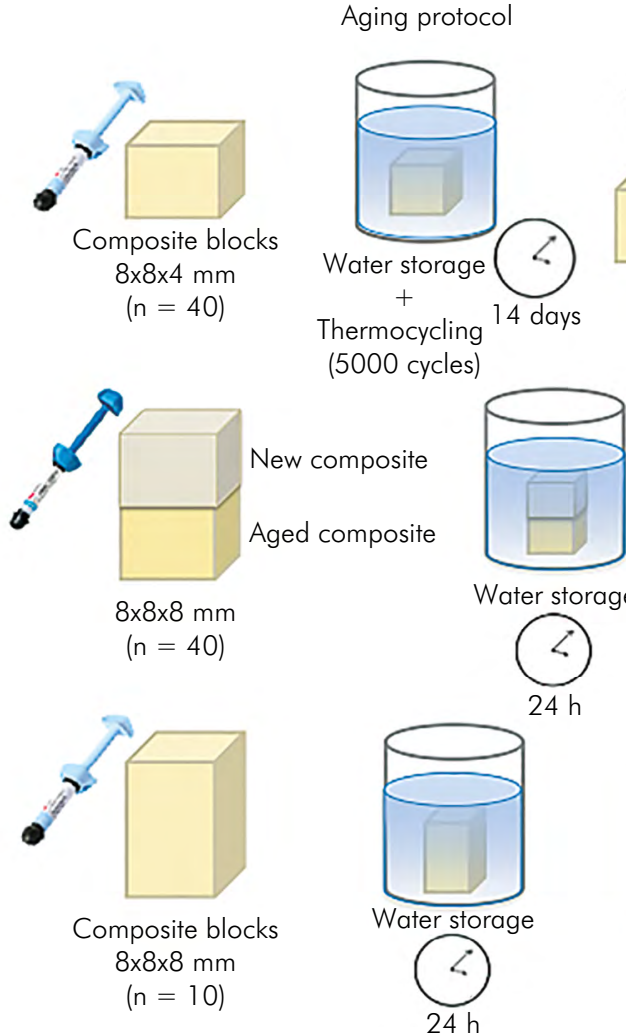

Water storage

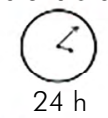
$24 \mathrm{~h}$

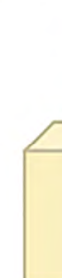

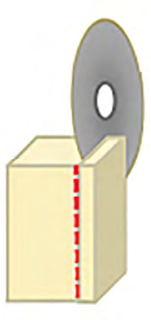

Surface treatments
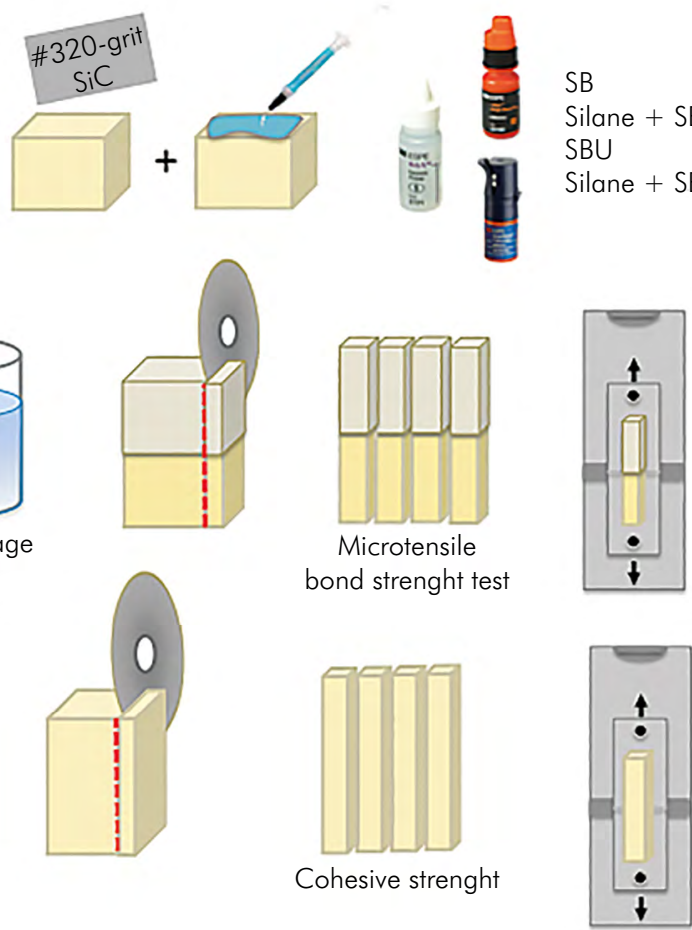

SB: Adper Single Bond Plus, SBU: Scotchbond Universal Adhesive.

Figure. Experimental design of the study. 
Table 2. The microtensile bond strength means (MPa), standard deviations, and distribution of the failure mode for all experimental groups

\begin{tabular}{lccccc}
\hline & Bond strength & $\begin{array}{c}\text { \% in relation to } \\
\text { cohesive strength } \\
\text { values }\end{array}$ & & \multicolumn{3}{c}{ Failure mode } \\
\cline { 5 - 6 } & & $39.3 \%$ & Adhesive & $\begin{array}{c}\text { Cohesive } \\
\text { (old composite) }\end{array}$ & $\begin{array}{c}\text { Cohesive } \\
\text { (new composite) }\end{array}$ \\
\hline Adper Single Bond Plus & $27.5 \pm 10.5^{\mathrm{B}}$ & $30.6 \%$ & $4.9 \%$ & $4.5 \%$ \\
Silane + Adper Single Bond Plus & $35.7 \pm 3.7^{\mathrm{B}}$ & $51.1 \%$ & $87.7 \%$ & $7.3 \%$ & $5.0 \%$ \\
Scotchbond Universal Adhesive & $38.7 \pm 10.5^{\mathrm{B}}$ & $55.4 \%$ & $93.7 \%$ & $4.3 \%$ & $2.0 \%$ \\
Silane + Scotchbond Universal Adhesive & $46.0 \pm 8.4^{\mathrm{B}}$ & $65.8 \%$ & $83.0 \%$ & $10.0 \%$ & $7.0 \%$ \\
Cohesive strength - Reference group & $69.9 \pm 17.8^{\mathrm{A}}$ & & & &
\end{tabular}

*Different capital superscript letters indicate statistically significance differences between cohesive strength values and bond strength values of the repaired groups $(p<0.05)$.

Main factors "adhesive system" ( $p=0.002)$ and "silane coupling agent" ( $p=0.03)$ were statistically significant. On the other hand, the cross-product interaction "adhesive system $v$ s. silane coupling agent" was not statistically significant ( $p=0.85$ ).

The $\mu$ TBS values were higher for the silane-containing universal adhesive compared to the two-step etch-and-rinse adhesive system. Previous silane application improved the repair bond strength, irrespective of the adhesive system (Table 3 ).

\section{Discussion}

Both silane coupling agents and adhesive systems appeared to establish an adequate bond strength between the aged composite and the new composite. ${ }^{12}$ Considering the "universal application" idea behind these contemporary all-in-one adhesives, use of a silane-containing universal adhesive for composite repair would simplify the clinical protocol, thereby reducing chair time and operator errors. In the current study, silane-containing universal adhesive used with previous acid etching produced higher repair bond strength values compared to the two-step etch-and-rinse adhesive system.

Composite surfaces aged in vitro show superficial dissolution and increased surface roughness, which may contribute to mechanical entanglement of the adhesive systems. ${ }^{21}$ Additionally, Scotchbond Universal Adhesive contains 10-MDP, a functional monomer that can chemically bond to zirconia surface..$^{22}$ Considering the zirconia content of fillers in Z350 XT, the 10-MDP monomer may help to promote repair bond strength by providing additional chemical bonding. However, applying silane separately improved the repair bond strength, irrespective of the adhesive. Therefore, the tested hypothesis was rejected.

Removing the superficial layer from an old composite and roughening it with a diamond bur is necessary to obtain micromechanical retention. In laboratory studies, the standardized surface roughness is obtained using 320-grit silicon carbide grinding paper, which simulates the roughness obtained with a medium diamond bur. ${ }^{19,20}$ This physical treatment can dissolve or remove the polymer matrix covering the glass fibers or particles and create a state where silane coupling agents can interact with silica. Degradation of dental composites during storage can also break filler-polymer bonds, allowing for surface loss of glass particles. ${ }^{23}$ Although there is currently no consensus on an aging method that can completely imitate clinical conditions, the current study chose to age the composite resin using water storage for $14 \mathrm{~d}$ followed by 5,000 thermocycles. ${ }^{19}$

Silane coupling agents promote chemical bonding by forming siloxane bonds between silicate-containing

Table 3. The microtensile bond strength means $(\mathrm{MPa})$ and respective standard deviations considering the main factors

\begin{tabular}{lccc}
\hline Adhesive system & Bond strength & Silane coupling agent & Bond strength \\
\hline Adper Single Bond Plus & $31.6 \pm 10.0^{\mathrm{B}}$ & With silane & $40.8^{\mathrm{A}} \pm 9.8^{\mathrm{A}}$ \\
Scotchbond Universal Adhesive & $42.3 \pm 7.4^{\mathrm{A}}$ & Without silane $^{\mathrm{B}}$ & $33.1 \pm 9.3^{\mathrm{B}}$ \\
\hline
\end{tabular}

Different capital superscript letters indicate statistically significant differences for each factor, separately $(p<0.05)$. 
filler particles exposed on the repair surface and the resin matrix of a fresh resin layer. ${ }^{24}$ Additionally, silanes have greater surface wettability, facilitating adhesive penetration into surface defects ${ }^{25}$ and improving the repair bond strength. Scotchbond Universal adhesive contains prehydrolyzed silane that the manufacturer claims is stable up to at least one year in storage. However, the amount of silane in its composition is not reported by the manufacturer and may be not sufficient to improve the repair bond strength.

A previous study ${ }^{17}$ also found that silane surface treatment improved $\mu \mathrm{TBS}$ of a silane-containing universal adhesive before composite placement. Conversely, a other study ${ }^{18}$ reported that silane-containing universal adhesive alone was as effective as any combination of silane and adhesive. This contradictory finding may be due to methodological differences related to bond strength test (microshear versus microtensile) and type of composite resin (nanofilled versus nanohybrid). Differently of the composite resin Z350XT, Filtek Supreme Ultra Restorative composite (3M Oral Care) contains silane-treated ceramic, silane-treated silica and silane-treated zirconia. It has been reported that incorporation of silanized filler particles in the resin matrix improves the physical and mechanical properties of resin composites in terms of mechanical strength and hydrolytic stability ${ }^{26}$ and it may have an influence in the surface treatment for repair.

Repair bond strength is measured as the maximum force prior to specimen fracture. If a large percentage of specimens are cohesively fractured, few conclusions can be drawn regarding repair bond strength because bond strength is usually lower than cohesive strength. The majority of failures observed in the current study across all experimental groups were adhesive. We used the cohesive strength of composites that were not aged as a reference for the desired or optimal repair strength. The cohesive strength of new material is unrealistic in aged specimens because composites gradually lose strength as they age. ${ }^{19}$ The repair bond strength for each substrate material ranged from 39.3 to $65.8 \%$ of the cohesive strength of the reference group. The silane paired with silane-containing universal adhesive repair protocol resulted in the bond strength that was closest to the cohesive strength of the reference group. Thus, the probability of failures at the composite-repair interface such as fractures could be minimized. It should be emphasized that the repair procedure for direct composites involves a dental structure in most clinical scenarios. Scotchbond Universal Adhesive shows satisfactory bonding to enamel and dentin. ${ }^{27}$ Therefore, bonding between a silane-containing universal adhesive and dental substrate, which usually involves repairing a restoration, could help minimize the need for prior silane application to the surface composite. The likelihood of obtaining a chemical bond to a composite substrate slowly decreases over time due to post-curing and water uptake. This leads to hydrolysis of available double bonds and few carboxyl groups for chemical bonding to a new composite. ${ }^{28}$ Furthermore, one-bottle prehydrolyzed silane solutions, such as RelyX Ceramic Primer, have a relatively short shelf life and gradually become less reactive after the bottle has been opened, thereby preventing optimal adhesion ${ }^{29}$. Future studies evaluating the use of silane as a pretreatment or incorporating it with the adhesive to enhance repair durability are necessary in order to recommend a universally applicable repair protocol. It is important to note that the current results are limited to the materials used in this study and may not apply to other silane-containing universal adhesives.

\section{Conclusions}

Under these study conditions, use of a universal silane-containing adhesive improved the repair bond strength of composite resin compared to a two-step etch-and-rinse adhesive. However, it still required prior application of a silane agent for best direct composite resin repair outcomes.

\section{References}

1. Demarco FF, Collares K, Correa MB, Cenci MS, Moraes RR, Opdam NJ. Should my composite restorations last forever? Why are they failing? Braz Oral Res. 2017 Aug;31 suppl 1:e56. https://doi.org/10.1590/1807-3107bor-2017.vol31.0056 
2. Chisini LA, Collares K, Cademartori MG, Oliveira LJ, Conde MC, Demarco FF, et al. Restorations in primary teeth: a systematic review on survival and reasons for failures. Int J Paediatr Dent. 2018 Mar;28(2):123-39. https://doi.org/10.1111/ipd.12346

3. Gordan VV, Riley JL 3rd, Rindal DB, QvistV, Fellows JL, Dilbone DA, et al. Repair or replacement of restorations: A prospective cohort study by dentists in The National Dental Practice-Based Research Network. J Am Dent Assoc. 2015 Dec;146(12):895-903. https://doi.org/10.1016/j.adaj.2015.05.017

4. Wilson N, Lynch CD, Brunton PA, Hickel R, Meyer-Lueckel H, Gurgan S, et al. Criteria for the Replacement of restorations: academy of operative dentistry European Section. Oper Dent. 2016 Sep;41 S7:S48-57. https://doi.org/10.2341/15-058-O PMID:27689930

5. Sharif MO, Catleugh M, Merry A, Tickle M, Dunne SM, Brunton P, et al. Replacement versus repair of defective restorations in adults: resin composite. Cochrane Database Syst Rev. 2014 Feb;(2):CD005971. https://doi.org/10.1002/14651858.CD005971.pub3

6. Sharif MO, Fedorowicz Z, Tickle M, Brunton PA. Repair or replacement of restorations: do we accept built in obsolescence or do we improve the evidence? Br Dent J. 2010 Aug;209(4):171-4. https://doi.org/10.1038/sj.bdj.2010.722

7. Elderton RJ. Clinical studies concerning re-restoration of teeth. Adv Dent Res. 1990 Jun;4(1):4-9. https://doi.org/10.1177/08959374900040010701

8. Kanzow P, Wiegand A, Göstemeyer G, Schwendicke F. Understanding the management and teaching of dental restoration repair: systematic review and meta-analysis of surveys. J Dent. 2018 Feb;69:1-21. https://doi.org/10.1016/i.jdent.2017.09.010

9. Ruiz LF, Nicoloso GF, Franzon R, Lenzi TL, de Araujo FB, Casagrande L. Repair increases the survival of failed primary teeth restorations in high-caries risk children: a university-based retrospective study. Clin Oral Investig. 2020 Jan;24(1):71-7. https://doi.org/10.1007/s00784-019-02899-9

10. Casagrande L, Laske M, Bronkhorst EM, Huysmans MC, Opdam NJ. Repair may increase survival of direct posterior restorations - A practice based study. J Dent. 2017 Sep;64:30-6. https://doi.org/10.1016/i.jdent.2017.06.002

11. Kanzow P, Wiegand A, Schwendicke F, Göstemeyer G. Same, same, but different? A systematic review of protocols for restoration repair. J Dent. 2019 Jul;86:1-16. https://doi.org/10.1016/i.jdent.2019.05.021

12. Valente LL, Sarkis-Onofre R, Gonçalves AP, Fernández E, Loomans B, Moraes RR. Repair bond strength of dental composites: systematic review and meta-analysis. Int J Adhes Adhes. 2016 Sep;69:15-26. https://doi.org/10.1016/i.ijadhadh.2016.03.020

13. Flury S, Dulla FA, Peutzfeldt A. Repair bond strength of resin composite to restorative materials after short- and long-term storage. Dent Mater. 2019 Sep;35(9):1205-13. https://doi.org/10.1016/i.dental.2019.05.008

14. Cuevas-Suárez CE, Nakanishi L, Isolan CP, Ribeiro JS, Moreira AG, Piva E. Repair bond strength of bulk-fill resin composite: effect of different adhesive protocols. Dent Mater J. 2020 Mar;39(2):236-41. https://doi.org/10.4012/dmj.2018-291

15. Loomans BA, Cardoso MV, Roeters FJ, Opdam NJ, De Munck J, Huysmans MC, et al. Is there one optimal repair technique for all composites? Dent Mater. 2011 Jul;27(7):701-9. https://doi.org/10.1016/i.dental.2011.03.013

16. Matinlinna JP, Lung CY, Tsoi JK. Silane adhesion mechanism in dental applications and surface treatments: a review. Dent Mater. 2018 Jan;34(1):13-28. https://doi.org/10.1016/i.dental.2017.09.002

17. Eliasson ST, Dahl JE. Effect of curing and silanizing on composite repair bond strength using an improved micro-tensile test method. Acta Biomater Odontol Scand. 2017 Mar;3(1):21-9. https://doi.org/10.1080/23337931.2017.1301211

18. Fornazari IA, Wille I, Meda EM, Brum RT, Souza EM. Effect of surface treatment, silane, and universal adhesive on microshear bond strength of nanofilled composite repairs. Oper Dent. 2017 Jul/Aug;42(4):367-74. https://doi.org/10.2341/16-259-L

19. Eliasson ST, Tibballs J, Dahl JE. Effect of different surface treatments and adhesives on repair bond strength of resin composites after one and 12 months of storage using an improved microtensile test method. Oper Dent. 2014 Sep-Oct;39(5):E206-16. https://doi.org/10.2341/12-429-L

20. Altinci P, Mutluay M, Tezvergil-Mutluay A. Repair bond strength of nanohybrid composite resins with a universal adhesive. Acta Biomater Odontol Scand. 2017 Dec;4(1):10-9. https://doi.org/10.1080/23337931.2017.1412262

21. Ferracane JL. Hygroscopicand hydrolytic effects in dental polymernetworks. DentMater. 2006 Mar;22(3):211-22. https://doi.org/10.1016/i.dental.2005.05.005

22. Valente LL, Silva MF, Fonseca AS, Münchow EA, Isolan CP, Moraes RR. Effect of diamond bur grit size on composite repair. J Adhes Dent. 2015 Jun;17(3):257-63.

23. Baena E, Vignolo V, Fuentes MV, Ceballos L. Influence of repair procedure on composite-to-composite microtensile bond strength. Am J Dent. 2015 Oct;28(5):255-60. PMID:26714342

24. Çakir NN, Demirbuga S, Balkaya H, Karadaş M. Bonding performance of universal adhesives on composite repairs, with or without silane application. J Conserv Dent. 2018 May-Jun;21(3):263-8. https://doi.org/10.4103/JCD.JCD_11_18

25. Brendeke J, Ozcan M. Effect of physicochemical aging conditions on the composite-composite repair bond strength. J Adhes Dent. 2007 Aug;9(4):399-406.

26. Lin CT, Lee SY, Keh ES, Dong DR, Huang HM, Shih YH. Influence of silanization and filler fraction on aged dental composites. J Oral Rehabil. 2000 Nov;27(11):919-26. https://doi.org/10.1046/i.1365-2842.2000.00573.x

27. Cuevas-Suárez CE, Rosa WL, Lund RG, Silva AF, Piva E. Bonding performance of universal adhesives: an updated systematic review and meta-analysis. J Adhes Dent. 2019;21(1):7-26.

28. Lagouvardos PE, Pissis P, Kyritsis A, Daoukaki D. Water sorption and water-induced molecular mobility in dental composite resins. J Mater Sci Mater Med. 2003 Sep;14(9):753-9. https://doi.org/10.1023/A:1025080103857

29. Lung CY, Matinlinna JP. Aspects of silane coupling agents and surface conditioning in dentistry: an overview. Dent Mater. 2012 May;28(5):46777. https://doi.org/10.1016/i.dental.2012.02.009 